\title{
Proceedings of the 128th Meeting of the Society of British Neurological Surgeons, University of Central Lancashire, Preston, 10-12 April 1996
}

SHOULD NEUROSURGEONS CARE FOR PATIENTS WITH MALIGNANT BRAIN TUMOURS?

C Davis, U Mukherjee, S Vhora. Royal Preston Hospital, Preston, UK

Introduction-The results of treatment of malignant glioma remain depressing. The recent Calman report on cancer services does not consider brain tumours.

Materials and methods-Since 1987 the Royal Preston Hospital has embarked on specialisation within the neurosurgical unit There is a weekly neuro-oncology clinic for patients with primary malignant brain tumours. With the introduction of this budgetless service there has been a rise in demand. The service networks into the community via general practitioners, cancer nurses, and local hospices and is primarily run by the nursing and secretarial staff on a daily basis. A withdrawal of social work from hospital has been difficult to overcome. Two hundred and twelve patients have been treated with over 1200 outpatient attendances and more than 1000 courses of palliative chemotherapy have been given.

Results-Patients with malignant brain tumours need constant support and have special problems distinct from other terminal cancer patients. The neurosurgeon is in a good position to meet the continuing care needs of this group of patients. The financial and time consequences of this care were discussed.

Conclusion-Proper neuro-oncology services are required in the United Kingdom; neurosurgeons sitting in large ivory towers (with few exceptions) should take heed of this unmet need.

MANAGEMENT OF OPTIC PATHWAY GLIOMAS

PO Byrne, G O'Reilly, CL Mallucci, AD Hockley, M Stevens, J Mann, D Spooner. Department of Neurosurgery and Departments of Radiotherapy and Oncology, Childrens' Hospital, Birmingham, UK

Objective-An analysis of outcome related to treatment in patients with optic pathway gliomas.

Design-Retrospective study of case notes of children in the West Midlands identified from the Regional Tumour Registry.

Patients-Fifty two patients up to the age of 14 years treated for optic pathway gliomas between 1958 and 1994 by combinations of surgery, radiotherapy, and chemotherapy, all with histological review.

Results and conclusions-Most patients were treated by surgery or radiotherapy, or a combination of both. Late recurrences occurred in both groups. There was no sta- tistical difference in five year survival in relation to presence or absence of neurofibromatosis, and no statistical difference in five year survival related to tumour site in the optic nerve, chiasm, or optic tract. Radiotherapy was more effective in preserving sight and did confer a five year surviva advantage $(P<0.03)$ over surgery alone. Radiotherapy was, however, associated with endocrine complications, particularly in children treated under the age of three years. Treatment is valuable to preserve sight early rather than late, with surgery alone being good for optic nerve lesions when there is no prospect of sight recovery. The role of chemotherapy requires further assessment.

CAN THE MORBIDITY FOR THE SURGICAL MANAGEMENT OF CHILDHOOD CRANIOPHARYNGIOMA BE PREDICTED?

RD Hayward, CJ De Vile, WFJ Harkness, BE Kendall, KE Watkins. Medical and Neurosciences Units, Institute of Child Health and Great Ormond Street Hospital for Children NHS Trust, London, UK

Objective and methods-Seventy five children treated for craniopharyngioma between 1973 and 1994 were studied to demonstrate which pre and intraoperative factors were indicative of a poor outcome as defined by a quantitative assessment of morbidity. This involved both a retrospective review of clinical details and cranial imaging in all but 10 patients (who were studied prospectively) and a follow up "study assessment" in 66 survivors performed over the past two years. As part of the second, 63 subjects had MRI which included a three dimensional volume acquisition sequence from 1.5 to 19.2 years after initial surgery.

Results-Predictors of high morbidity included severe hydrocephalus, intraoperative adverse events, and young age $(\leqslant 5$ years) at presentation. Predictors of increased hypothalamic morbidity included whether symptoms of hypothalamic disturbance were already established at diagnosis, height of the tumour in the mid-line, and whether adherent tumour was removed from the region of the hypothalamus at operation. Tumour size, young age, and severe hydrocephalus were predictors of tumour recurrence whereas complete tumour resection (as determined by postoperative neuroimaging) and radiotherapy given electively after subtotal excision were less likely to be associated with recurrent disease.

Conclusion-A protocol has been designed that allows an individualised and more flexible treatment approach in an attempt to provide both long term tumour control and the lowest morbidity for children with craniopharyngioma.
PAEDIATRIC MENINGEAL TUMOURS: A REVIEW OF 21 CASES

CL Mallucci, S Parkes, J Powell, M Stevens, AR Walsh, AD Hockley. Departments of Neurosurgery and Oncology, Birmingham Children's Hospital, Birmingham, UK

Objective-To study the clinical aspects, pathology, and outcome of these rare childhood tumours.

Design-A retrospective analysis of meningeal tumours in children under 16, treated in the West Midlands between 1957 and 1993.

Subjects-Sixteen patients had meningiomas (median age 13 years, nine male, seven female) and five had malignant meningeal tumours (median age 3 years, one male, four female). Three had neurofibromatosis.

Outcome measures-Histology was available in 20 patients. Median clinical follow up was 18 years. Actuarial survival analysis was performed by the Kaplan-Meier method.

Results-There were 19 supratentorial tumours (five intraventricular), one tentorial, and one cervical. Surgical excision was complete in 12 patients, incomplete in six, and two were biopsied. The remaining patient had a radiological diagnosis only. All five patients in the malignant undifferentiated group died (median survival five months) compared to an actuarial five and 15 year survival of the meningiomas of $79 \%$ $(P=0.0001)$. Complete excision and absence of focal neurological deficit at presentation were favourable prognostic indicators.

Conclusions-There is an affinity for paediatric meningeal tumours to have a supratentorial origin and intraventricular location. The female preponderance found in adults is not evident in children. Incomplete surgical excision or focal neurological deficit at presentation were associated with poor outcome.

CLINICAL PRESENTATION AND MANAGEMENT OF 100 INFANTS WITH OCCIPITAL PLAGIOCEPHALY

IK Pople, RA Sanford, MS Muhlbauer. Department of Neurosurgery, Frenchay Hospital, Bristol, UK and SemmesMurphey Clinic and Le Bonnheur Children's Medical Center, Memphis, Tennessee, USA

Objective-To determine results of both surgical and non-surgical management of infants presenting with occipital plagiocephaly.

Design-Retrospective analysis of clinical features, treatment, and outcome of all infants presenting with a flattened occiput. 
Subjects-One hundred consecutive infants with occipital plagiocephaly.

Outcome measures-Cosmetic appearance six months after presentation.

Results-18 infants who showed obvious progression of their deformity or radiological signs of fusion of the lambdoid suture had surgical resection of one or both lambdoid sutures and the remaining 82 infants were treated non-surgically with physiotherapy and advice on sleeping position. Of the 18 surgical cases nine were found at operation to have true lambdoid synostosis, seven had pronounced internal ridging of the lambdoid suture, and two had an open suture. All parents of the 82 children treated non-surgically were satisfied with their child's cosmetic appearance: $63 \%$ showed improvement in their plagiocephaly, and $37 \%$ stabilised. There were no surgical complications in the 18 operative cases, who all had a good cosmetic result.

Conclusion-Most infants referred to neurosurgeons with occipital plagiocephaly can be successfully managed non-surgically. A small proportion of cases with radiological signs of fusion or pronounced internal ridging of the suture may benefit from surgery.

NEUROENDOSCOPY IN THE MANAGEMENT OF CHILDHOOD HYDROCEPHALUS

J Punt, M Vloeberghs. Departments of Neurosurgery and Child Health, University Hospital, Nottingham, UK

Objective-To review the value of neuroendoscopy in the management of childhood hydrocephalus.

Design-Retrospective review of clinical records.

Patients-Sixty seven patients aged 9 weeks to 22 years who underwent neuroendoscopy primarily for the management of hydrocephalus between 1992 and 1995.

Outcome measures-Relief of symptomatic hydrocephalus. Need for subsequent ventricular shunt.

Results-Neuroendoscopy was performed using either flexible or rigid endoscopes. Patients underwent the procedures by way of primary treatment of hydrocephalus (28); management of shunt complications (20); treatment of intracranial cysts (eight); management of tumoral hydrocephalus (nine); or for evacuation of intraventricular haematomas (two). Third ventriculostomy was the most frequent procedure (32) of which 12 failed, mostly in children aged under six months. There were only three other failures. In 10 cases of intraventricular or paraventricular tumour, biopsy was successful in seven and partial excision in one, in addition to relieving the hydrocephalus. There were no significant complications. Conclusions-Neuroendoscopy has a definite place in the management of childhood hydrocephalus, although it is often unsuccessful in the first six months of life. It should be considered as an option in cases of blocked ventricular shunt.

SPATIAL MODULATION OF MAGNETISATION IN THE STUDY OF CEREBROSPINAL FLUID DYNAMICS: IMPLICATIONS ON THE PATHOPHYSIOLOGY OF SYRINGOMYELIA

DE Sakas, CM Tolias, DJ Beale, SC Wayte, B Williams. Walsgrave Hospital, Coventry and the Midlands Centre for Neurosurgery and Neurology, Smethwick, UK

Spatial modulation of magnetisation (SPAMM) is an MRI technique, first introduced as a method of assessing motion in 1989. ${ }^{1}$ The technique has been modified, ${ }^{2}$ and used to measure CSF flow in the prepontine region and both dorsal and ventral to the spinal cord at the $\mathrm{C} 1-\mathrm{C} 2$ vertebral level, in patients and healthy volunteers. The presence of flow inside the syrinx has been assessed in patients with syringomyelia before and after craniocervical decompression. At the C1-C2 vertebral level, in patients with hindbrain hernia and syringomyelia the preoperative CSF velocities were found to be reduced by $26 \%$ ventral to, and $73 \%$ dorsal to the spinal cord, when compared with those of healthy volunteers $(2.8(0.3) \mathrm{cm} / \mathrm{s}$ and $2.4(0.2) \mathrm{cm} / \mathrm{s}$ respectively). After craniocervical surgical decompression, SPAMM showed that CSF velocities increased by $94 \%$ ventral to the cord, and $42 \%$ dorsal to the cord. Prepontine velocities followed similar patterns.

SPAMM is a reliable quantitative method of assessing CSF motion under normal and pathological conditions. The data provide an insight into the CSF dynamics responsible for the pathogenesis of syringomyelia. SPAMM may help to clarify some of the questions about the mechanism maintaining and expanding the syrinx.

1 Axel L, Dougherty D. MR imaging of motion with spatial modulation of magnetization. with spatial modulation

2 Wayte SC, Redpath TW, Beale DJ. MRI of pulsatile CSF flow by SPAMM. Br $f$ Radiology 1992;65:495-501.

UK SHUNT REGISTRY AND UK SHUNT EVALUATION UNIT: A PROGRESS REPORT

JD Pickard, C O'Kane, HK Richards, M Czosnyka, Z Czosnyka, H Whitehouse, L Fahie. Academic Neurosurgical Unit, Addenbrooke's Hospital, Hills Road, Cambridge, UK

Objective-Eighty per cent of CSF shunts fail by 12 years. The progress of the UK Shunt Registry and the UK Evaluation Unit towards the identification of substandard valves, audit of standards of care, and risk stratification has been assessed.

Methods-The registry started with a pilot phase in eight centres to create a report form compatible with the Patient Analysis Tracking System software; 1192 forms have been returned for March-October 1995 from 37 of the 56 centres identified as undertaking shunt surgery. The laboratory was established in November 1993 based on a comprehensive test protocol.

Results-Quality and quantity of reporting varied between centres with 222 revisions (mean revision rate per centre of $18.6 \%$ (95\% CI 16.4-20.8)) and wide variation in practice. Eighty per cent of the valves were supplied by one manufacturer. Testing nine of the commonest valves reveals features of relevance to surgeons that are not always adequately described in the manufacturers' product information.

Conclusion-Implementation of both registry and laboratory promise to place the management of hydrocephalus on a more rational basis but greater motivation in individual centres will be required to ensure the suc- cess of the registry.

Acknowledgements and conflict of interestFunding from the Medical Devices Agency and East Anglian Regional Audit Committee is independent of any manufacturer.

PAIN AFTER MICROVASCULAR DECOMPRESSION OF THE TRIGEMINAL NERVE

MK Hunn, B West, LT Dunn, PR Eldridge, JB Miles. Walton Centre for Neurology and Neurosurgery, Rice Lane, Liverpool, UK

Objective-To analyse the cause of pain in the face, continuing after microvascular decompression of the trigeminal nerve (MVD) for trigeminal neuralgia (TGN).

Methods-Assessment of the outcome after MVD, with interview and additional analysis of those still complaining of pain in the face. Repeat magnetic resonance tomoangiography (MRTA)

Results-One hundred and thirteen patients underwent MVD and 109 replied to questionnaire. Thirty nine $(35 \%)$ had some pain continuing in the face. In 10 patients this was TGN, in five minor TGN, and in six a mixed pain syndrome. Seven patients had definite dysaesthetic pain, all previously having undergone radiofrequency coagulation.

Repeat MRTA was undertaken on 25 patients, some with and some without TGN and will be analysed blindly retrospectively.

Two patients have been cured of their TGN by repeat MVD and two, with negative MRTA, had successful neurotomy at reexploration.

Three patients had temporary continuing pain which cleared spontaneously.

Conclusion-Facial pain present after MVD can take various forms, is capable of analysis especially by the use of MRTA, and can benefit from a range of different treatments including repeat MVD.

TRIGEMINAL NEURALGIA: REVIEW OF 58 CASES TREATED BY MICROVASCULAR DECOMPRESSION USING IVALON SPONGE

J Patel, H Wilson, $\mathbf{P}$ Kane, $\mathbf{F}$ Nath. Middlesbrough General Hospital Neurosurgical Department, Middlesbrough, UK

Introduction-The results of microvascular decompression (MVD) for trigeminal neuralgia (TGN) are inconsistent because of different variables. This study reviewed one surgeon's results over 10 years who operated on patients of all ages and used Ivalon sponge alone.

Patients and methods-Data were collated by patient interview, case notes review, and postal questionnaires. Between 1986-95, 58 patients (42 F:16 M) with age ranges from 35-88 (mean 67) years had a MVD through a retromastoid microcraniectomy. A pathological vessel was recorded as definite, subjacent (indefinite), or absent. The incidence of pain relief, its rate of recurrence, and the number of complications were noted.

Results-Patients were followed up from three months to nine years (mean 4.5 years). Unrelated deaths occurred in nine patients. There were 15 patients who had previous alternative treatments. Vascular anomalies were definite in 44 patients and subjacent in 
11. Immediate and complete pain relief was obtained in 54 patients. Recurrence occurred in four patients. Facial hypoaesthesia, although subjective and minimal was present in 15 patients. Other complications were CSF leakage (four), corneal ulceration (two), facial herpes zoster (two), reduced hearing (one), and superficial wound infection (one).

Conclusion-Pain relief occurred in $93 \%$ of the patients with a recurrence rate of $7 \%$. However, the incidence of facial hypoaesthesia was high (26\%). It is possible that other factors are important in the treatment of TGN and warrant further study.

THE VALUE OF PREOPERATIVE EMBOLISATION OF CRANIAL MENINGIOMAS

SN Bhagwati, M Dhawal, D Dadhich. Department of Neurosurgery, Bombay Hospital, Bombay, India

Meningiomas are vascular tumours. Often during their excision, there is copious blood loss despite the use of hypotension. Besides the need to replace a large quantity of blood, excessive bleeding precludes good visualisation of the operative site and renders critical dissection of neurovascular structures difficult. Preoperative endovascular embolisation of these tumours was therefore used to reduce their blood supply and facilitate their excision

In 186 patients preoperative embolisation was performed 24-48 hours before surgery. Predominant supply from the external carotid artery was from the meningeal arteries. Even falcine meningiomas seemed to receive appreciable vascularity from the meningeal arteries. Gelfoam particles were routinely used after superselective cannulation of the feeders from the external carotid artery.

Evaluation of efficacy of embolisation in reduction of the tumour was based on comparison of the vascularity of the tumour as seen on preembolisation and postembolisation angiographic pictures as well as on the quantity of blood transfused during the operation. There was a significant reduction in blood loss during surgery in $90 \%$ of cases with only external carotid artery supply. Some of these tumours looked pale, almost infarcted at the time of excision. Significant reduction in blood loss was found even in $30 \%$ of cases that had dual blood supply

It is considered that preoperative endovascular embolisation with gelfoam particles is a safe and useful tool that effectively reduces the vascularity of meningiomas supplied by the external carotid circulation and enables these meningiomas to be excised more completely, with greater safety and with much reduced blood loss.

HIGH DOSE APROTININ REDUCES BLOOD LOSS IN SURGERY FOR INTRACRANIAL MENINGIOMA

JD Palmer, D Simpson, D Francis, JL Francis, JD Pickard, F Iannotti. Clinical Neurological Sciences and University Department of Haematology, Southampton University Hospitals and Neurosurgery Unit, Addenbrooke's Hospital, Cambridge, UK

Objectives-To measure the efficacy of high dose aprotinin in reducing peroperative bleeding in neurosurgery. The high concentration of tPA in meningioma may be responsible for induction of fibrinolysis and consequent bleeding disorders. Aprotinin (Trasylolß) reduces fibrinolysis and protects platelet function in addition to its anticoagulant properties.

Design-Randomised, double blind, placebo controlled, two centre, study.

Subjects-Fifty six patients (aged 30-84 years) undergoing surgery for intracranial meningioma. Patients previously exposed to aprotinin, or those who had received aspirin, NSAIDs, or anticoagulants in the two weeks before surgery were excluded. A loading dose of $30000 \mathrm{KIU}$ aprotinin $/ \mathrm{kg}$, or saline placebo, was followed either by an infusion of $10000 \mathrm{KIU} / \mathrm{kg} / \mathrm{hour}$ or equivalent amount of saline during surgery.

Outcome measures-Operative blood loss, amount of blood transfused, operation time, and screening for DVT at day 5 .

Results-Median blood loss was $1202 \mathrm{ml}$ in the placebo and $576 \mathrm{ml}$ in the treatment group, corresponding to $52 \%$ reduction $(\mathrm{P}=$ 0.03 , Wilcoxon two sample test). The mean transfusion requirement was 1.3 (SD 2.0) units in the aprotinin group compared with $2 \cdot 2$ (SD 3.4 ) units in the controls, a reduction of $41 \%$. The presence of DVT was demonstrated with duplex scanning in $31 \%$ of the control group compared with only $17 \%$ of the treated patients (NS). Operating time was shortened by aprotinin (NS).

Conclusions-Aprotinin reduces surgical bleeding and blood transfusion and may also reduce the risk of DVT.

\section{This study was funded by Bayer UK}

IS THE PRESENT MODEL OF CLINICAL DIRECTORSHIP IN THE NEUROSCIENCES ACCEPTABLE?

RJ Nelson, YP Clarke. Department of Neurosurgery, Frenchay Hospital, Bristol, UK

Objective-To determine the workload, training, and management support of clinical directors of neuroscience units and to obtain their views on the present model of clinical directorship.

Design-Structured postal questionnaire supplemented by telephone enquiry and a residential seminar.

Subjects-Thirty nine neuroscience units were approached and complete data were received from $26(66.6 \%)$ clinical directors. Main results-The mean age of the clinical directors was 49 (range 38-60) and the average length of time spent as a consultant was 12 (range 1-27) years. In most cases (53\%) two sessions had been allocated to the clinical directorship but only four clinical directors had reduced their clinical sessions. Fifty per cent of clinical directors had had no formal training in financial management, $73 \%$ in NHS business and contracting, $65 \%$ in personnel management, and $88 \%$ in statutory and legal responsibilities. Only $2 \%$ of clinical directors had the full time support of a manager and secretary.

Conclusions-The present generation of clinical directors have accepted a substantial additional managerial workload, often with little formal training and inadequate managerial support; $53 \%$ of the clinical directors concluded that the present model of clinical directorship is unsustainable in the long term.
"SAFE NEUROSURGERY": HOW ARE WE DOING THREE YEARS ON?

N Buxton, J McMullan, P Byrne, B White. Department of Neurosurgery, University Hospital, Queen's Medical Centre, Nottingham, UK

Objective-To review how neurosurgical services in the United Kingdom and Eire have developed since publication of Safe Neurosurgery three years ago.

Design-A postal survey of all neurosurgical units listed in Safe Neurosurgery with telephone follow up, to identify staff levels, working patterns, bed numbers, ITU, HDU, theatre, and paediatric provision, before Safe Neurosurgery, now and planned.

Results-A $100 \%$ response was obtained. The number of consultants has increased from 132.5 in 1993 to 158.0 now, and 165 within the next year, compared with the recommended $170 \cdot 5$. There has been a modest increase in juniors' numbers (11 higher trainees and nine senior house officers).

During the past three years there has been a reduction in national bed numbers from 1550 to 1476 (a shortfall of 381 compared with the absolute minimum recommended by Safe Neurosurgery) and a loss of five dedicated neurosurgical ITU beds from 126 to 121 (against a recommended 248 required). A fall in HDU beds from 143 to 125 has also occurred whereas there has been a modest increase in theatre facilities from 63.5 to 67 (85 required). The larger units have made most gains.

Conclusions-Safe Neurosurgery is a seminal document which sets the minimum resource standards required for the delivery of safe neurosurgical care into the next century. Some improvements towards these standards have been made with regard to consultant numbers (driven largely by the juniors' hours imperative) but there remains a nationwide shortfall of provision and pronounced local variation.

MAGNETIC RESONANCE ANGIOGRAPHY (MRA) IN ANEURYSMAL SURGERY

S Vhora, AJ Keogh, WJ Gunawardena, CMA Coutinho. Royal Preston Hospital, Preston, UK

Objective-MR images were used to plan surgery without recourse to intra-arterial digital subtraction angiography (DSA). MRA is reliable in detecting ruptured aneurysms in the acute phase of illness in a proportion of patients.

Methods-All patients presenting with a subarachnoid haemorrhage over a two year period from 1993 to 1995 were considered for MR studies as part of their investigation. If aneurysms were detected, the images obtained were used to plan surgery at various supratentorial sites. ${ }^{2}$

Results-Of some 200 patients presenting with a subarachnoid haemorrhage 130 were submitted for MR studies. In 55 of these patients aneurysms were demonstrated with the images being obtained considered to be satisfactory to plan early surgery. There were 21 men and 34 women with an age range of 23 to 79 years and a mean of 50.5 years. Thirty eight of the 55 patients underwent subsequent DSA studies to ensure that no other lesion had been missed.

Conclusion-MR studies-non-invasive and avoiding radiation-can be rapidly obtained 
in many patients presenting with a subarachnoid haemorrhage with the images sufficient to plan early surgery if clinically appropriate.

1 Sankhla S, Gunawardena WJ, Coutinho CMA, Keogh AJ. Magnetic resonance angiography in the management of aneurysmal subarachnoid haemorrhage: a study of 51 cases. $\mathcal{F}$ Neurol Neurosurg Psychiatry 1995;58:118-19.

2 Keogh AJ, Sankhla SK. Magnetic resonance angiography for anterior midline aneurysms. Br $\mathcal{F}$ Neurosurg 1996;10:143-7.

MAGNETIC RESONANCE SPECTROSCOPY AND DIFFUSION WEIGHTED IMAGING IN THE ASSESSMENT OF CEREBRAL ISCHAEMIA AFTER SUBARACHNOID HAEMORRHAGE

Z Domingo, J Bradley, B Rajagopalan. MRC Clinical Magnetic Resonance Unit, John Radcliffe Hospital, Oxford, UK

New MRI techniques and the clinical use of magnetic resonance spectroscopy (MRS) hold the promise of early non-invasive recognition of the ischaemic complications of vasospasm enabling prompt treatment with improved outcome. The MRI/MRS changes seen in patients represent a heterogeneous group of pathophysiological mechanisms making interpretation difficult.

The application of endothelin 1 (ET1) to the MCA in rats results in focal low flow ischaemia more accurately reflecting the clinical situation compared with conventional models of focal cerebral ischaemia. ${ }^{12}$ The T2 and diffusion weighted images (DWI), in addition to the changes in ${ }^{31} \mathrm{P}$ and ' $\mathrm{H}$ spectra, were investigated in this model of vasospasm. Fifteen rats were studied.

${ }^{31} \mathrm{P}$ MRS showed an immediate drop in $\mathrm{pH}$ to 6.54 and a rise in the $\mathrm{Pi}: \mathrm{PCr}$ and $\mathrm{Pi}$ :ATP ratios. There was a concomitant rise in the lactate peak on ${ }^{1} \mathrm{H}$ MRS. Over the ensuing two hours the $\mathrm{pH}$ and metabolite ratios returned to normal and the lactate peak disappeared. Throughout this period T2 images remained normal. DWI showed early and persistent changes.

In conclusion, conventional $\mathrm{T} 2$ imaging is inadequate to detect the early changes of low flow ischaemia. The return to normal of the ${ }^{31} \mathrm{P}$ and ${ }^{1} \mathrm{H}$ spectra do not imply a reduction in ischaemia but ongoing flow to the hemisphere. The addition of DWI and ${ }^{31} \mathrm{P}$ and ${ }^{1} \mathrm{H}$ MRS to conventional imaging may enable more accurate assessment of the metabolic state of the brain in patients with vasospasm and assessment of the efficacy of proposed treatment.

1 Macrae IM, Robinson MJ, Graham DI, Reid J, McCulloch J. Endothelin-1-induced reductions in cerebral blood flow, dose dependency, time course, and neuropathological consequences. $\mathcal{F}$ Cereb Blood Flow Metab 1993;13:276-84.

2 Sharkey J, Ritchie IM, Kelly PAT. Perivascular microapplication of endothelin-1: a new
model of focal cerebral ischaemia in the rat. $f$ Cereb Blood Flow Metab 1993;13:865-71.

A PROSPECTIVE AUDIT OF ANEURYSMAI SUBARACHNOID HAEMORRHAGE COMPARING EARLY AND LATE SURGERY

PJA Hutchinson, H Seeley, PJ Kirkpatrick. Academic Neurosurgical Unit, Addenbrooke's Hospital, Hills Road, Cambridge, UK

Introduction-The results of an ongoing prospective audit of outcome of patients with aneurysmal subarachnoid haemorrhage
(SAH) are reported. The effect of timing of surgery on the three month Glasgow outcome scale score (GOS) and the length of neurosurgical stay was sought.

Methods-A standard proforma has been used since September 1992 for recording the demographic and clinical details of all patients with SAH admitted to the East Anglian Regional Neurosurgical Unit. Data was independently collected by the Clinical Audit Department.

Results-Demography: between September 1992 and December 1995, 420 patients were admitted with SAH (mean age 53.1 years; range $16-89$ years; $59 \%$ women). The mean time from bleed to hospital admission was 2.4 days, and 259 patients underwent clipping of their aneurysm at a mean time of $6 \cdot 1$ days postbleed.

Comparison of early and late surgery: $59 \%$ of the operated patients underwent early (0-4 days) surgery (mean 2.3 days) and $41 \%$ late ( $>4$ days) surgery (mean 10.0 days). Both groups were well matched for demographic features and severity of SAH. The outcome at three months was favourable (GOS1 and GOS2) in $84 \%$ of the early group and $85 \%$ of the late group. The operative mortality rate at three months was $7 \%$ and $9 \%$ respectively. The duration of stay was significantly shorter $(95 \%$ CI P < $0.001)$ for those undergoing early surgery (mean time 16.6 days) compared with those undergoing late surgery (23.2 days). In this unit this corresponds to a saving of 759 inpatient hospital days per year.

Conclusion-Surgical outcome is independent of the timing of aneurysm surgery, but those who undergo late surgery have a longer inpatient stay.

\section{DELAYED ANEURYSM SURGERY: A} REAPPRAISAL

RS Maurice-Williams, J Wadley. The Royal Free Hospital and School of Medicine, London, UK

Delayed aneurysm surgery, once standard practice, is now followed by only a minority of neurosurgeons. The outcome of such a policy in 400 consecutive patients with ruptured aneurysms treated over a 14 year period was analysed. Despite an "open door" admissions policy, admitting all patients immediately on referral, only $56 \%$ arrived within 24 hours of the ictus $(69 \%$ within 72 hours). Of the very early admissions $33 \%$ were in grades 4 and 5 . Surgery was generally delayed for 8 to 10 days in patients in grades 1 and 2; for higher grade patients often for longer until their condition was stable. Two hundred and eighty seven patients $(72 \%)$ underwent surgery, $93 \%$ on day 8 or later $(78 \%$ on day 11 or later). Outcome was assessed at one year. For all patients $68 \%$ were in Glasgow outcome scale grade 1 while $26 \%$ died. Of the operated patients $88 \%$ were in Glasgow outcome scale grade 1 while $5 \%$ died (30 day surgical mortality was $3.5 \%$ ). Fifty one patients $(12.8 \%)$ rebled, 30 in the first 10 days. Rebleeding was distributed evenly in time over the first two weeks. Eighty four patients experienced non-haemorrhagic deterioration (NHD) all but three within 10 days; NHD peaked at days 4 to 9 . Thirty three patients died of rebleeding and 16 of NHD but only 12 of the patients who died from rebleeding were fit for operation at any time and might have been considered for early surgery. Two of these patients died so soon after admission that surgery could not have been performed. The theoretical advantages of delayed as against early surgery lead to the conclusion that it is doubtful whether the timing of surgery has any significant effect on management outcome in line with the conclusions of the Cooperative Study.

PRELIMINARY EVALUATION OF LIPOPROTEIN

(A) AS A BIOLOGICAL MARKER FOR PRERUPTURED FAMILIAL INTRACRANIAL ANEURYSMS

GA Roberts, C Bolger, JP Phillips. Department of Neurosurgery, Beaumont Hospital, Dublin, Ireland.

Background-Management of patients with familial intracranial aneurysms (ICAs) will be greatly improved when a biological marker becomes available to screen for asymptomatic cases. Raised serum lipoprotein (a) $[\mathrm{Lp}(\mathrm{a})]$, an independent risk factor for atherogenesis, has been demonstrated in both sporadic and familial ICAs.

Objectives - To assess the degree of correlation between raised $\mathrm{Lp}$ (a) concentrations and the occurrence of asymptomatic ICAs in first degree relatives of patients with familial aneurysmal subarachnoid haemorrhage.

Design-Three families were studied, each with two or more members having had aneurysmal subarachnoid haemorrhage. Asymptomatic first degree relatives were screened by random serum $\mathrm{Lp}$ (a) sampling and cerebral angiography.

Patients-A total of 24 subjects, 11 males and 13 females, with mean age 43.9 years. Results-Ten subjects with asymptomatic ICAs were detected and all received treatment. Nine of these had a $\mathrm{Lp}$ (a) concentration above $45 \mathrm{mg} / \mathrm{dl}$. Fourteen subjects did not have aneurysms visible on angiography. Serum Lp(a) was less than $45 \mathrm{mg} / \mathrm{dl}$ in eight of these 14. Six subjects had a $\mathrm{Lp}(\mathrm{a})$ above $45 \mathrm{mg} / \mathrm{dl}$ with negative angiography. Four of these were in the second or third decade of life. One 20 year old woman with $\mathrm{Lp}(\mathrm{a})$ of $80 \mathrm{mg} / \mathrm{dl}$ had what seemed intraoperatively to be an aneurysm in evolution. Mean $\mathrm{Lp}$ (a) was $58.6 \mathrm{mg} / \mathrm{dl}$ in patients with ICAs and $39.7 \mathrm{mg} / \mathrm{dl}$ in those without demonstrable aneurysms.

Conclusions - Lp(a) may be important in the pathogenesis of ICAs and may be a potentia biological marker. Follow up studies are required on angiographically negative subjects. We have begun a genetic case-control study to establish if particular apoprotein (a) gene polymorphisms can be correlated with the occurrence of intracranial aneurysms.

OUTCOME OF SUBARACHNOID

HAEMORRHAGE: AN ANALYSIS OF SURGICAL VARIABLES AND COGNITIVE AND EMOTIONAL SEQUELAE RELATED TO SPECT

RAC Jones, E Berry, CGH West. Departments of Neurosurgery and Behavioural Medicine, Hope Hospital, Salford, UK

This prospective study evaluated outcome after aneurysmal haemorrhage and surgery with respect to medical factors, cognitive function, psychological distress and regional cerebral blood flow. 
For the evaluation of cognitive outcome, 48 patients were compared with a control group, matched for age, sex, and premorbid IQ. A significant difference between the two groups was found on measures of memory, with a significant deterioration in intellectual ability in the patient sample, irrespective of aneurysm site.

None of the medical factors investigated contributed to the significant differences found; in particular, temporary vessel occlusion and aneurysmal rupture during surgery did not themselves adversely affect outcome. Seventeen $(33 \%)$ patients showed abnormalities on SPECT. No significant difference in cognitive function (on any measure) was found between patients with normal and abnormal SPECT.

A proportion of patients showed significant levels of distress on psychometric measures of depression (BDI), general health (GHQ 28) and anxiety (STAIT, TRAIT). No significan difference in cognitive outcome was found between those with normal and elevated Beck depression inventory scores but there was a tendency for those with impaired memory to show higher levels of anxiety. It is concluded that the deleterious effects of subarachnoid haemorrhage are due to the impact of the initial haemorrhage, not the site of rupture or surgical variables.

A COMPARISON OF READ CODES WITH THE ICD10 AND OPCS-4 CODING SYSTEMS FOR NEUROSURGERY

KS O'Neill, SA Cudlip, JP Grieve, BA Bell Atkinson Morley's Hospital, London, UK

Introduction-With advances in information technology the need for an electronic language to handle clinical data has become paramount. To this end the NHS executive has acquired and developed the Read codes but we still depend on the ICD-10 and OPCS-4 systems for purposes of audit and central returns.

Objectives-To demonstrate the use of READ, ICD-10, and OPCS-4 codes in clinical practice, comparing their comprehensiveness, specificity, and accuracy as coding systems.

Design-A computerised patient database system has been developed incorporating Read codes for diagnosis and procedures. In addition cross matched ICD-10 diagnostic codes and OPCS-4 procedure codes are recorded. One hundred diagnostic and 100 operative terms were chosen and coded with each system comparing specificity.

Results-For $100 \%$ of clinical core terms a separate and specific Read code was identified. For ICD-10 and OPCS-4 some terms shared the same code and several codes had multiple Read code cross matches. In comparison ICD-10 was only $42 \%$ as specific as Read overall, the worst being spinal diagnoses $(16.3 \%)$, the best tumour diagnoses $(98 \%)$. OPCS -4 were overall $79 \%$ as specific as Read, the worst being spinal procedures $(59 \%)$ and the best ventricular shunting procedures $(100 \%)$

Conclusion-The Read codes are a comprehensive system encompassing almost any clinical terminology. They are a superset of ICD-10 and OPCS-4, being more accurate and specific. Within an appropriate patient database system and ergonomic infrastructure they would be an invaluable clinical data collection tool.
THE MODIFIED TRANSBASAL APPROACH: SURGICAL INDICATIONS AND LIMITATIONS

G Neil-Dwyer, DA Lang, BT Evans, S Honeybul, JS Gill. Wessex Neurological Centre and Department of Oral MaxilloFacial Surgery, Southampton University Hospitals, Southampton, UK

Objective-To determine the surgical morbidity, applications, and limitations of the modified transbasal approach.

Design-Patients with central skull base pathology requiring operation were identified as suitable for the approach which incorporates a bifrontal craniotomy, extended orbito-frontal bandeau and en bloc ethmoidectomy-where appropriate olfaction can be preserved by removing the ethmoid block in continuity with the basal dura and a cuff of nasal mucosa.

Patients-Ten patients aged from 10-73; six females. Each had benign pathology in the juxtasellar/jugum sphenoidale/clivus region. Results-Six patients had a meningioma, two chordomas, one craniopharyngioma, and one fibrous dysplasia.

In each case excellent access to the central skull base was obtained with minimal frontal lobe retraction.

There were no complications directly due to the access used. Three patients developed temporary diabetes insipidus. One patien died at three months. One patient is severely disabled (craniopharyngioma). The remaining patients (eight) are moderately disabled or have made a good recovery (minimum six months). Excellent recovery of vision has occurred in those patients presenting with visual failure.

Conclusion-The approach is technically demanding and a multidisciplinary team is essential. The advantages include improved access with better visualisation of the pathology and the relevant neurovascular anatomy.

ENDOSCOPIC MANAGEMENT OF CEREBROSPINAL FLUID RHINORRHOEA: THE NOTTINGHAM EXPERIENCE

RGM Hughes, NS Jones, IJA Robertson. Department of Neurosurgery, Queen's Medical Centre, Nottingham, UK

Objective-To review the outcome of endoscopic management of CSF rhinorrhoea. Design-Retrospective review of patients who have undergone endoscopic repair of CSF rhinorrhoea.

Patients-There were 15 patients (aged 45 to 62) with verified CSF rhinorrhoea due to various pathologies (1993-5).

Outcome measures-Cessation of CSF rhinorrhoea.

Results - There were 15 patients who underwent endoscopic repair of defects in the cribiform plate, fovea ethmoidalis, and sphenoidal sinus using free grafts of turbinate or fascia lata. Fourteen out of 15 patients had complete cessation of rhinorrhoea at 1-30 months follow up. The only patient to have persistent rhinorrhoea after attempted endoscopic repair underwent a second procedure which also failed. No significant complications were seen.

Conclusion-In many cases CSF rhinorrhoea can be treated successfully by endoscopic surgery, with low morbidity.
MANAGEMENT OF MENINGIOMAS OF THE CAVERNOUS SINUS

MG O’Sullivan, K Park, H van Loveren, JM Tew. Department of Clinical Neurosciences, Western General Hospital, Edinburgh, UK and University of Cincinnati, Cincinnati, Ohio, USA

The optimal management for patients with cavernous sinus meningioma is controversial. In this retrospective review, 39 patients underwent surgical treatment for this condition including 27 women and 12 men ranging in age from 24 to 73 years (median 48 years). Completeness of tumour resection, cranial nerve morbidity, general morbidity, and the long term outcome were studied. The cavernous internal carotid artery (ICA) was partially encased in 15 patients, totally encased in 11 patients, and narrowed by tumour in 13 patients. Of eight patients who had a complete tumour resection, seven had partial encasement of the ICA. Of 31 patients who had a subtotal resection, 11 underwent postoperative radiotherapy. There were no deaths in the series. Morbidity was $17 \cdot 9 \%$ for cranial nerves controlling extraocular motor function. Trigeminal nerve function did not improve after surgical treatment. The median follow up period was two years (range 6 months to 5.3 years). Symptomatic and radiographic recurrence occurred in two patients who had complete tumour resection and in two patients who had subtotal resection. Based on these findings and a review of the literature, it is concluded that: $(a)$ the resectability of meningiomas of the cavernous sinus depends on the degree of ICA involvement; (b) total excision of holocavernous meningiomas is rarely achieved; (c) cranial nerve morbidity is significant; and (d) subtotal excision with or without postoperative radiotherapy is an effective short term oncological sirategy.

HEAD INJURY IN THE SEVERELY INJURED:

LONG TERM FOLLOW UP IN 157 PATIENTS

LT Dunn, $M$ Patterson, DA Boot, RV Jeffreys. Walton Centre for Neurology and Neurosurgery, Liverpool and Department of Orthopaedics, Warrington Hospital NHS Trust, Warrington, UK

Objective-To assess the degree and cause of disability persisting after multiple injury.

Design-Prospective review of cohort of multiply injured patients (ISS $>15$ ) four to five years after hospital discharge.

Patients-A total of 212 adult patients admitted between 1 May 1989 and 30 April 1990 with ISS > 15 who left hospital alive (part of a group of 1088 victims of severe trauma identified in the Mersey Region and North Wales during the above period).

Outcome measures-Patient interview and examination by specialist nurse practitioner. Assessment using the Bull disability scale and Glasgow outcome scale.

Results - Of 212 patients who left hospital alive 157 were traced, interviewed, and examined. Of this group 80 were assessed as having had a significant head injury as one of their multiple injuries (AIS $2-5$ ). $74 \%$ of these made a good recovery, $18 \%$ had moderate disability, and $8 \%$ severe disability as assessed by the Glasgow outcome scale. Very severe disability on the Bull disability 
scale was always due to brain or spinal cord injury. By contrast $63 \%$ of disability in the severe disability group and $72 \%$ of disability in the moderate disability group was assessed as due to musculoskeletal injury affecting the limbs, spine, or pelvis. Many patients expressed dissatisfaction with the level of care they received after hospital discharge.

Conclusions-In multiply injured patients severe disability is almost always due to brain or spinal cord injury. In less severely disabled patients musculoskeletal injuries play a much bigger part. There is widespread dissatisfaction with the level of post hospital care and rehabilitation in this group of patients.

EARLY COMBINED TREATMENT OF COMPLEX CRANIOMAXILLOFACIAL TRAUMA

D Oluoch-Olunya, JC Beirne, D O'Brien, M Fitzgibbon, C Pidgeon. Department of Neurosurgery, Trauma Audit, Beaumont Hospital, Dublin, and Department of Maxillofacial Surgery, St James's Hospital, Dublin, Ireland

Objective-To present the initial results of early combined one stage neurosurgical and maxillofacial operative management of complex craniomaxillofacial trauma.

Design-Forty patients treated over a two year period were retrospectively studied with follow up ranging from three months to two years.

Results-Thirty four were adults and six were in the paediatric age group with an age range of 2 years to 57 years. The aetiology was road traffic accident in 24 cases, assault four, horse riding four, industrial accident four, fall three, and shotgun injury one. Five patients had other associated injuries. Fifteen patients had frontonaso-orbital fractures, nine fronto-orbital, and 16 had Le Fort II/III type fractures with a concomitant dural tear or involvement of the posterior wall of the frontal sinus. The time range to definitive surgery from the initial trauma was three hours to 45 days, mean 4.8 days. The intraoperative time was a mean of 6.5 hours. One operation required the facial reconstruction to be performed as a secondary procedure due to excessive blood loss. There were no postoperative deaths. One patient developed a late onset CSF leak and one patient required removal of his frontoorbital reconstruction due to infection.

Conclusions-In selected patients early combined treatment of complex craniomaxillofacial fractures is feasible with a low postoperative morbidity.

BIFRONTAL DECOMPRESSIVE CRANIECTOMY IN THE MANAGEMENT OF DIFFUSE MALIGNANT POSTTRAUMATIC CEREBRAL OEDEMA

RS Polin, D Mohan, JA Jane, LH Pobereskin. Departments of Neurosurgery, University of Virginia, Charlottesville, Virginia, USA and Derriford Hospital, Plymouth, Devon, UK

Objective-To examine the efficacy of decompressive frontal craniectomy in the management of diffuse malignant post-traumatic cerebral oedema.

Design-Comparison of Glasgow outcome scale score in patients receiving decompressive craniectomy with controls matched by
Glasgow coma score, intracranial pressure, age, and sex.

Subjects-There were 35 patients who underwent the procedure and 92 matched controls taken from the traumatic coma data bank.

Outcome measures-Glasgow outcome scale score at three months in the controls and at acute care hospital discharge in the subjects was used as the primary outcome measure.

Results-The patients undergoing decompressive craniectomy and their controls were remarkably similar in terms of admission Glasgow coma score $(5.6 v 5.7)$ and maximum preoperative sustained intracranial pressure (34.9 torr $v 33.0$ torr). Whereas the overall rate of favourable outcomes (good recovery or moderate disability) in the surgical patients was only $37 \%$ (13 of 35 ), multivariate logistic regression analysis showed a statistical advantage of the operated patients over controls $(P=0.029)$. Surgery was also effective in reducing preoperative raised intracranial pressure $(P=0.0003)$.

Conclusions-Operative management of diffuse malignant post-traumatic cerebral oedema in the absence of mass lesions improved outcomes over medical treatment alone. Bifrontal craniectomy provides a viable management alternative in the treatment of these difficult patients.

TRAUMATIC INTRACRANIAL HAEMATOMA: CAN WE DO EVEN BETTER?

K Seex, G Teasdale, L Murray, D Gentleman. Department of Neurosurgery, Southern General Hospital, Glasgow, UK

Objective-Despite decreasing after the introduction of guidelines, mortality after a traumatic haematoma remains high; the aim was to discover opportunities for further improvements.

Design, subjects, and outcome measures-(1) retrospective review of the records of 115 patients with a poor outcome to determine conformity of management with guidelines. (2) prospective analysis of 50 patients to investigate the relation of the place of CT (DGH or NSU), and the timing of surgery.

Results-(1) compared with the 1984 guidelines, errors in preneurosurgical management were detected in 56 cases. These included delayed referral to neurosurgery in 33 of 51 admitted to a district general hospital. Application of the 1990 extended guidelines, which encouraged CT in general hospitals, could have led to earlier detection of a haematoma in the other 18 cases. (2) in patients who had CT before referral, the median time from first arrival at hospital to operation was 425 minutes, but was 290 minutes in those referred to neurosurgery for CT. However, this difference arose from patients considered to be clinically "static", not those deteriorating.

Conclusions-Complete adherence to the extended guidelines could improve the management and outcome of $64 \%$ of patients who still have a poor outcome but this will depend on general hospitals ensuring rapid access of appropriate cases to CT.

ANALYSIS OF SPINAL TRAUMA MANAGED IN HULL

F Selmi, B Mathew. Hull Royal Infirmary, Anlaby Road, Hull, UK
Objective-Can management of spinal fractures be undertaken successfully in small neurosurgical units?

Design-A retrospective review of the last 100 cases found to have a spinal fracture. Period of study was 23 months, from January 1994 to November 1995. Clinical presentation, treatment, and outcome were analysed using records and $x$ ray films.

Patients - Of 100 patients, 59 were male and the median age was 44.4 (range 4 to 83 years). Injuries were due to falls (69), road traffic accidents (20), and other causes (11). Results-Ninety four were single level. Lumbar vertebrae were involved in 51 , thoracic in 32 , and cervical in $27 . \mathrm{Ll}$ was the commonest level involved. In the cervica region C2 fractures of various types predominated. Seventy were compression fractures, 11 burst fractures, four fracture dislocations, and eight were $\mathrm{C} 2$ fractures. ${ }^{1}$ All had local pain, seven had focal neurological signs, and three were quadriparetic. Seventy seven patients were treated conservatively, Halovest stabilisation was used in four patients. Nineteen had a fusion using wire and bone, transpedicular screws, and Hartshill rectangle.

Outcome-Conservative group: 74 discharged, one kyphus, one death. Operated group: 15 discharged, three minimal backache, one persistent neurological deformity. None had progression of neurological deficit or deformity.

Conclusion-Good results can be achieved in small neurosurgical units undertaking surgery for spinal trauma.

1 Dennis F. The three column spine and its significance in the classification of acute significance in the classification of acute
thoracolumbar spinal injuries. Spine 1983;8: thoracoll

2 Jacobs RR, Asher MA, Snider RK. Thoracolumbar spinal injuries: a comparative study of recumbent and operative treatment in 100 patients. Spine 1980;5:463-77.

DEVELOPMENT OF THE MYELOPATHY FUNCTIONAL DISABILITY INDEX: A STEP TOWARDS OBJECTIVE ANALYSIS OF THE RESULTS OF CERVICAL SPINE SURGERY

ATH Casey, HA Crockard. The National Hospital for Neurology and Neurosurgery, London, UK

Objectives-To be able to objectively measure disability in rheumatoid arthritis complicated by cervical myelopathy.

Design - The responses to the Stanford health assessment questionnaire disability index were recorded from 250 consecutive patients (group 1) referred to the unit for spinal surgery. Using principal components analysis the questionnaire was reduced from 20 questions to 10 questions. In the second part of the study, the results of the questionnaire for those patients undergoing surgery from the original group of 250 patients were analysed with respect to outcome.

Results - The reduction in the number of questions resulted in no significant loss of information, reliability (internal consistency Cronbach's $\alpha=0.968$ ) or sensitivity. The new scale, the myelopathy disability index, measures only one dimension (Eigen value 6.97) and is hopefully more finely tuned to measuring disability in these myelopathic patients. When administered to the 194 patients undergoing cervical spine surgery (group 2) the myelopathy disability index was an accurate predictor of neurological 
and functional outcome, as well as survival after surgery ( $p<0.0001)$.

Summary-The myelopathy disability index provides a much needed objective and reliable means of assessing disability in patients with rheumatoid involvement of the cervical spine and also in predicting outcome after surgical intervention. It also provides information for both the patient and surgeon alike, on what to realistically expect from surgery. Its adoption will facilitate comparisons between different forms of surgical intervention.

MANAGEMENT OF UPPER CERVICAL CORD COMPRESSION IN MUCOPOLYSACCHARIDOSIS

DP O'Brien, J Thorne, JE Wraith, RA Cowie. Willink Biochemical Genetics Unit and Department of Neurosurgery, Royal Manchester Children's Hospital and Hope Hospital, Salford, Manchester, UK

Objectives-Craniovertebral anomalies commonly occur in many inherited disorders. The mucopolysaccharidoses (MPS) are a group of seven inherited biochemical disorders (types I-VII) caused by a deficiency of specific lysosomal enzymes. They are characterised by skeletal changes, mental retardation, and visceral involvement. Cervical myelopathy may be due to $\mathrm{C} 1-\mathrm{C} 2$ subluxation and/or dural thickening. Our experience of surgery in patients with MPS with upper cervical cord compression is described.

Design-Retrospective analysis of experience of patients with MPS with emphasis on presentation, neuroradiology, surgical management, and final functional outcome.

Subjects-Thirteen patients with MPS were reviewed (type II/Hunter's syndrome, three; type IV/Morquio's syndrome, eight; type VI/Maroteaux-lamy syndrome, one). The male to female ratio was $6: 7$ and the age range was 2-29 years (mean 10.3 years; median 9.0 years). All patients required extensive preoperative neuroradiological ear, nose, and throat, and cardiorespiratory evaluation.

Results-Eleven cases presented with cervical myelopathy, two cases presented with asymptomatic $\mathrm{C} 1-\mathrm{C} 2$ subluxation. Elective tracheostomy was necessary in three cases. Craniocervical decompression alone $(n=3$ all were Hunter's syndrome and all required duraplasties), craniocervical fusion alone ( $=7$ ), or both decompression with fusion (
=3) were performed. There were two wound infections which responded to conservative treatment and there were no deaths in the series. All symptomatic patients ( $n=$ 11) made varying degrees of functional improvement.

Conclusion-Patients with MPS with either evidence of spinal cord compression or asymptomatic upper cervical subluxation, should be considered as candidates for spinal decompression or fusion surgery.

REOPERATIVE TRANSPHENOIDAL SURGERY FOR CUSHING'S DISEASE

AG Chenelle, LH Poberskin, ER Laws. Departments of Neurosurgery, University of Virginia, Charlottesville, Virginia, USA and Derriford Hospital, Plymouth, UK

Objective - To examine the success and complication rates in transphenoidal reoperation for recurrent/residual disease in patients with Cushing's disease.

Design-Retrospective review of over 2600 patients in one of the author's transphenoidal series to identify 29 patients with Cushing's disease undergoing reoperation after failed primary surgery. Success of the secondary procedure, complication rates, influence of prior radiation therapy, and long term success were analysed by retrospective chart review.

Patients-Twenty nine patients, two men and 27 women were identified as undergoing repeat transphenoidal surgery for Cushing's disease. Average age was 37 years. Eighteen patients had microadenomas and 11 had macroadenomas.

Outcome measures-Retrospective review of preoperative and postoperative cortisol concentrations were used to evaluate success of the operative procedure. Complications were analysed from the operative and perioperative notes.

Results-Fifty nine per cent of patients were operative cures. Three additional patients were cured with postoperative XRT. The surgical cure rate for microadenomas was $61 \%$. The surgical cure rate for macroadenomas was $55 \%$. In patients with prior radiation therapy the cure rate was $25 \%$. The major complication rate was $7 \%$.

Conclusion-Through a retrospective review of one author's series of salvage transphenoidal operations for Cushing's disease it has been shown that cure rates for both microadenomas and macroadenomas are better than expected and the complication rate, although higher than in primary transphenoidal operations, is acceptable.

TRANSPHENOIDAL SURGERY FOR ACROMEGALY

TPD Blackburn, R Sheaves, JAH Wass, GM Besser, F Afshar. Departments of Neurosurgery and Endocrinology, St Bartholomew's Hospital, London, UK

Objective-To determine the outcome of transphenoidal surgery for acromegaly. Mean $\mathrm{GH}<5 \mathrm{mU} / \mathrm{l}$ was used as the defining criterion for "cure", as recent evidence suggests that prognosis is optimised in this group. ${ }^{1}$

Design-A retrospective study of patients undergoing transphenoidal surgery for acromegaly at St Bartholomew's Hospital from 1985-95, operated on by the senior surgeon.

Patients-119 consecutive patients underwent transphenoidal surgery for acromegaly. Mean follow up period 3.2 years (range $0 \cdot 5-9 \cdot 5)$.

Outcome measures- $\mathrm{GH}$ concentrations are represented as a mean value from a four point day curve. Serum cortisol, TSH, T4, prolactin, LH, FSH, testosterone, or oestradiol, and urine and plasma osmolality were also measured.

Results-Postoperatively $43.7 \%$ of patients achieved a mean $\mathrm{GH}$ concentration $<5$ $\mathrm{mU} / 1$. Success rate was influenced by preoperative $\mathrm{GH}$ concentration, rising to $65 \%$ in those with preoperative concentrations $<20$ $\mathrm{mU} / 1$ but falling to $18 \%$ in those with $\mathrm{GH}$ concentrations $>100 \mathrm{mU} / 1$.

Tumour size also influenced outcome. $\mathrm{GH}$ concentrations $<5 \mathrm{mU} / 1$ was achieved in $61 \%$ of patients with microadenoma falling to $25 \%$ of patients with macroadenoma. Only one patient has shown evidence of recurrence after initially satisfying criteria for "cure". Perioperative mortality was zero. Two patients required surgical repair for CSF leak. There were three proved cases of meningitis.

Conclusions-Acromegaly has recurred in only one patient with a mean postoperative $\mathrm{GH}$ concentration $<5 \mathrm{mU} / 1$.

1 Bates AS, Van't Holt W, Jones JM, Clayton $R N$. An audit of outcome of treatment in acromegaly. $Q \mathcal{F}$ Med 1993;86:293-9. 